\title{
Pain Management in Mesothelioma
}

\author{
Nicholas MacLeod, ${ }^{1,2,}{ }^{*}$ Pal Klepstad, ${ }^{3,4}$ Marie Fallon, ${ }^{1,5}$ and Barry Laird ${ }^{1,2,5}$ \\ ${ }^{1}$ Edinburgh Cancer Research UK Centre, University of Edinburgh, Edinburgh, UK \\ ${ }^{2}$ Beatson West of Scotland Cancer Centre, Glasgow, UK \\ ${ }^{3}$ Department of Anaesthesiology and Emergency Medicine, St Olavs University Hospital, Trondheim, Norway \\ ${ }^{4}$ Department of Circulation and Medical Imaging, Norwegian University of Science and Technology (NTNU), Trondheim, Norway \\ ${ }^{5}$ Western General Hospital, Edinburgh, UK \\ "Corresponding author: Nicholas MacLeod, Edinburgh Cancer Research UK Centre, University of Edinburgh, Edinburgh, UK. Tel: +44-1413017679, Fax: +44-1413017604. E-mail: \\ nicholas.macleod@ggc.scot.nhs.uk
}

Received 2015 August 13; Accepted 2016 September 20.

\begin{abstract}
Pain is the most common symptom in malignant pleural mesothelioma and can be challenging to manage. This article discusses the pathophysiology of pain in mesothelioma and provides a case history to outline management options including medication, radiotherapy, chemotherapy and cordotomy.
\end{abstract}

Keywords: Pain, Malignant, Pleural, Mesothelioma

\section{Introduction}

Malignant pleural mesothelioma (MPM) is a rare cancer that originates from mesothelial cells, usually in the pleural lining of the chest. It is typically caused by exposure to asbestos and is traditionally associated with a long latency period, often in the region of $30-40$ years (1). The incidence is expected to peak in the next few years given the widespread use of asbestos in the second half of the last century. Unfortunately, there is no known cure for MPM and so the focus of management is often palliation of symptoms.

Pain is the most common symptom in MPM although fatigue and breathlessness are also highly prevalent (2). In MPM, pain usually affects the chest in keeping with the underlying disease. However the cause of chest pain in MPM is multifactorial. Pain may be due to direct tumour infiltration of the ribs, nerve roots, intercostal nerves, chest wall, or in some cases, due to the tumor invading the neurovascular bundle. In addition, in patients who undergo surgery, post thoracotomy pain is common (3). The pain associated with MPM is often more severe and difficult to treat than pain caused by lung cancer (4).

The pathophysiology of pain in MPM is generally a mixture of bone and neuropathic pain. Therefore, managing this pain can be notoriously difficult with multiple analgesics that target different pain mechanisms often being required. Unfortunately, patients may continue to suffer from severe pain despite multiple analgesics and so other options to consider for these patients include palliative radiotherapy, chemotherapy, neuroaxial pain therapy, and cordotomy. All these therapeutic options are discussed herein.

The difficulties of pain management in MPM are perhaps best illustrated via a case history.

\section{Case Presentation}

A 68-year-old man presented to his local hospital with severe right-sided chest pain and breathlessness. He had worked as an apprentice carpenter in the shipbuilding industry many years ago. A chest X-ray was performed that showed a right-sided pleural effusion and pleural thickening. A computerised tomography (CT) (Figure 1) scan, including a CT guided biopsy, was done with pathology confirming MPM of sarcomatoid type. Treatment options were discussed with the patient. Chemotherapy was discussed, but this was declined by the patient due to a low likelihood of benefit. The patient agreed that treatment would be symptomatic only. The gentleman described his pain as "stabbing and shooting", suggesting a neuropathic component. He also stated that it was severe in intensity. Prior to admission, he had been taking tramadol (400 mg daily) and gabapentin (300 mg three times daily). During his admission, he was commenced on $40 \mathrm{mg}$ of morphine sustained release tablets (every 12 hours), and $15 \mathrm{mg}$ of immediate release. Morphine tablets were given when needed for pain, and a lidocaine patch applied over the chest wall. Tramadol was discontinued. Pain continued to be problematic and when his morphine dose was escalated, he developed signs of opioid toxicity (muscle jerks and pseudohallucinations). He was therefore switched to oxycodone 
sustained release tablets ( $30 \mathrm{mg}$ twice daily). With this, his opioid toxicity improved, although it was still present. His gabapentin was increased to $600 \mathrm{mg}$ TDS and he remained on a lidocaine patch. Despite all this, his pain remained poorly controlled.

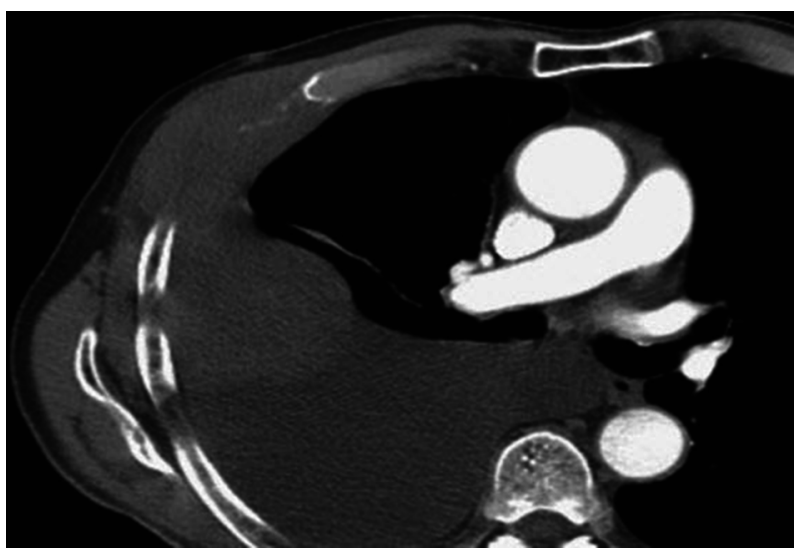

Figure 1. CT Scan Image Showing Mesothelioma Eroding Through the Chest Wall

At this point, he received radiotherapy with the aim of improving his pain. Twenty Gy in 5 fractions of radiotherapy were administered which brought about a temporary improvement in his pain, but six weeks after treatment, his pain was as severe as it was prior to his radiotherapy. Following this, he was considered for cordotomy, but unfortunately died before he was able to receive this procedure.

This case illustrates some of the difficulties patients with MPM face in terms of pain management. Despite multiple analgesics and palliative radiotherapy, his pain remained poorly controlled. Not all cases of MPM are as challenging as this. Given that the pain associated with MPM is often multifactorial, patients are often on several drugs, which work via different mechanisms of action. It is always important to weigh up the potential benefits of multiple analgesics against the possibility of cumulative toxicity, drug interactions and patient compliance. Commonly used medications and doses are detailed in Table 1. The key components of analgesic treatment are detailed below.

\section{Discussion}

\subsection{WHO Analgesic Ladder}

The world health organization (WHO) Analgesic ladder for cancer pain relief is recognised as the gold standard for treatment of cancer pain (Figure 2) and its principles has been continued in new guidelines $(5,6)$. It is easy to follow and can be applied to all types of pain, irrespective of aetiology. The first step on the ladder recommends using
Table 1. Drugs and Dose Ranges Commonly Used for pain Management in MPM

\begin{tabular}{|c|c|c|c|}
\hline Drug Name & $\begin{array}{c}\text { Type of } \\
\text { Analgesic }\end{array}$ & $\begin{array}{c}\text { Typical } \\
\text { Starting Dose }\end{array}$ & $\begin{array}{l}\text { Maximum } \\
\text { Daily Dose }\end{array}$ \\
\hline Paracetamol & Non Opioid & $1 \mathrm{~g}$ QDS & $4 \mathrm{~g}$ \\
\hline Diclofenac & Non Opioid & $50 \mathrm{mg}$ TDS & $150 \mathrm{mg}$ \\
\hline $\begin{array}{l}\text { Codeine } \\
\text { Phosphate }\end{array}$ & Weak Opioid & 30 - $60 \mathrm{mg}$ QDS & $240 \mathrm{mg}$ \\
\hline Morphine & Strong Opioid & $\begin{array}{c}10 \text { mg every } 4 \text { - } 6 \\
\text { hours }\end{array}$ & $\begin{array}{c}\text { No maximum } \\
\text { dose }\end{array}$ \\
\hline Oxycodone & Strong Opioid & $\begin{array}{c}5 \text { mg every } 4 \text { - } 6 \\
\text { hours }\end{array}$ & $\begin{array}{c}\text { No maximum } \\
\text { dose }\end{array}$ \\
\hline Hydromorphone & Strong Opioid & $\begin{array}{c}1.3 \text { mg every } 4-6 \\
\text { hours }\end{array}$ & $\begin{array}{c}\text { No maximum } \\
\text { dose }\end{array}$ \\
\hline Gabapentin & Adjuvant & $300 \mathrm{mg}$ OD & $3.6 \mathrm{~g}$ \\
\hline Pregabalin & Adjuvant & $75 \mathrm{mg}$ BD & $300 \mathrm{mg}$ \\
\hline Amitriptyline & Adjuvant & $10 \mathrm{mg}$ OD & $150 \mathrm{mg}$ \\
\hline Lidocaine & Adjuvant & $\begin{array}{l}5 \% \text { patch } 12 \\
\text { hours on, } 12 \\
\text { hours off }\end{array}$ & $\begin{array}{l}5 \% \text { patch } 12 \\
\text { hours on, } 12 \\
\text { hours off }\end{array}$ \\
\hline Fentanyl & Strong Opioid & $\begin{array}{c}12 \text { mcg per hour. } \\
\text { Change every } 72 \\
\text { hours }\end{array}$ & $\begin{array}{c}\text { No maximum } \\
\text { dose }\end{array}$ \\
\hline Ketamine & Adjuvant & $5 \mathrm{mg}$ BD & $\begin{array}{c}\text { No maximum } \\
\text { dose }\end{array}$ \\
\hline
\end{tabular}

Abbreviations: OD, once daily; BD, twice daily; TDS, three times daily; QDS, four times daily.

paracetamol or non-steroidal anti-inflammatories. If pain remains poorly controlled then patients should move on to step two, which involves the use of weak opioids such as codeine. However, most patients with pain from MPM will quickly move to step three of the ladder which recommends strong opioids plus or minus non-opioids or adjuvant analgesics. Therefore, step two may be skipped and a step three opioid used as the first line opioid (6). Morphine remains the most commonly used strong opioid although there are now a multitude of others available and often patients may try several different strong opioids in an attempt either to improve analgesic benefit/reduce toxicity or both (6).

\subsection{Opioids}

Given the severity of pain associated with MPM, the majority of patients will require opioid analgesia with morphine being the most commonly used. It comes in both immediate and sustained release preparations, which are equivalent in terms of analgesic benefit $(6,7)$. Immediate release preparations are by many favoured for initial dose titration and subsequent breakthrough analgesia while sustained release preparations are more typically saved for long term use (8). However, a direct titration using 


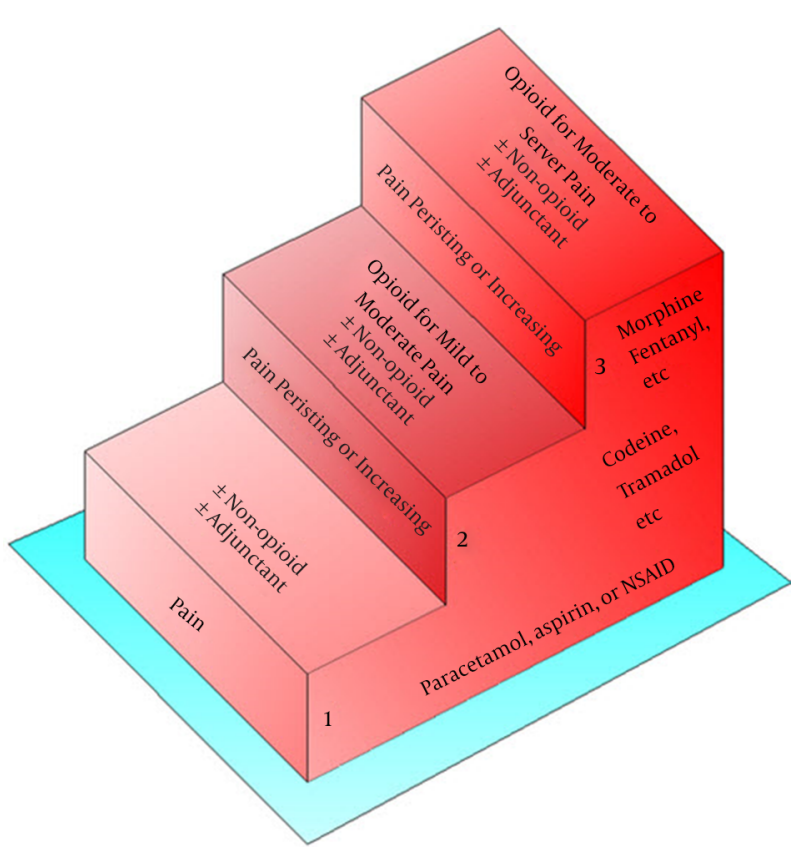

Figure 2. WHO Analgesic Ladder

sustained released opioids is equally feasible (7). Patients may require large quantities of opioids to help control their pain. It is not uncommon that on these large doses, patients develop toxicity with symptoms such as somnolence, myoclonic jerks, cognitive impairment and hallucinations. In these circumstances, most would favour a switch to another opioid such as oxycodone.

\subsection{Adjuvant Analgesics}

Adjuvant analgesics are drugs whose main indication is not analgesia, but they have analgesic properties. They are used in many types of pain including neuropathic pain. They can be used in combination with opioid analgesics or in isolation. However, in MPM, given the often severe intensity of pain, adjuvant analgesics are often used in combination with opioids (9). Given that there appears to be a significant neuropathic component to the pain in MPM, it is not surprising that these drugs are commonly used in these patients. There are several different types of adjuvant analgesics that can be helpful.

\subsection{Antidepressants}

A Cochrane review summarises the evidence for the use of antidepressants in non-malignant neuropathic pain (10). Tricyclic antidepressants are the most commonly used antidepressant in neuropathic pain. Amitriptyline has a marginally greater analgesic effect compared to other tricyclic antidepressants such as nortriptyline. Selective noradrenaline reuptake inhibitors (SNRI) such as duloxetine and venlafaxine may be of benefit in neuropathic pain, with duloxetine being favoured due to a better sideeffect profile (11).

\subsection{Anticonvulsants}

There is good evidence that anticonvulsants are effective in neuropathic pain (12). Gabapentin has been used in the treatment of neuropathic pain for many years and is recommended as a first line treatment (13). It is generally well tolerated though its dose limiting toxicity is usually somnolence. Pregabalin works in the same way as gabapentin and there is good evidence of its efficacy as an analgesic (14). A recent prospective, placebo controlled, randomised controlled trial compared pregabalin with gabapentin and amitriptyline for neuropathic cancer pain. The results suggested that all drugs were effective in relieving cancer-related neuropathic pain, but pregabalin was associated with the greatest reduction in pain scores (15).

\subsection{Other Analgesics}

\subsubsection{Ketamine}

There is some evidence supporting the benefit of ketamine in cancer pain (16). If symptoms exist which are suggestive of central wind-up such as pain on light touch or increased pain to any painful stimulus then ketamine may be helpful. Furthermore, ketamine may renew opioid response when opioid doses are being increased with reduced response.

\subsubsection{Topical Analgesics}

Topical analgesics can play a role in the treatment of pain in MPM with topical 5\% lidocaine patches being the most commonly used. The main benefit of these patches is the lack of systemic side effects with local skin irritation being the common side effect. Though there have been no prospective studies in cancer patients, a retrospective review from Australia looked at 97 patients treated with lidocaine 5\% patches and their results supported its use in post herpetic, post-surgical and cancer related neuropathic pain (17). The high potency $8 \%$ capsaicin topical patch has proven efficacy in post herpetic neuralgia (18). A single application of the patch for 30 - 60 minutes can result in pain relief for up to three months.

\subsubsection{Radiotherapy}

Many patients with MPM will continue to suffer from severe pain despite multiple analgesics. Therefore, radiotherapy is often considered for these patients. However, 
there is a dearth of evidence to support its use in any setting in MPM. Despite this, radiotherapy for pain relief is recommended in guidelines from the European Respiratory Society in collaboration with the European society of thoracic surgeons (19).

Perhaps the most convincing evidence in favour of radiotherapy to help pain comes from a study where 22 patients with MPM and pain were treated with hemi thoracic irradiation at a dose of 30 Gray in 10 fractions. Of the 19 patients assessable at three months, 13 had an improvement in their pain scores with no increase in their analgesic requirements though the median duration of response was only two months (20). A recent study looked at palliative radiotherapy at a dose of 36 Gray in 12 fractions (21). Radiotherapy was not given to the entire hemithorax, but instead was directed to the area that was felt to be causing the pain. This was because hemi-thoracic irradiation was felt to be too toxic. The study showed that pain improved in over $50 \%$ of patients two weeks after treatment though this was assessed via a retrospective review of case notes rather than prospectively. Interestingly, CT scans were performed in these patients two months after radiotherapy and a response rate of $43 \%$ was reported suggesting that, at an adequate dose, MPM may in fact be a radiosensitive disease.

\subsubsection{Chemotherapy}

Two phase III studies have shown a survival advantage in the region of two-three months for platinum/antifolate combination chemotherapy in MPM $(22,23)$. Quality of life data from one of these studies have been published (24). These data showed that pain scores remained constant throughout treatment. The authors concluded that, in a rapidly fatal disease such as MPM, stabilization of pain was a positive finding. However, the same data can also be interpreted as showing that chemotherapy does not improve pain control in MPM. Therefore, chemotherapy should be prescribed in this disease in an attempt to improve survival rather than to improve pain. If symptom improvement is the aim then other treatment options should be considered.

Although chemotherapy can be offered, a retrospective review of MPM patients showed that, of 156 patients diagnosed, only 54 were deemed of adequate performance status to be offered chemotherapy and ultimately, only 27 patients received chemotherapy. Therefore, this treatment is not widely used in this population (25).

\subsubsection{Epidural or Intrathecal Analgesia}

Epidural or intrathecal treatment, usually with a combination of an opioid and a local anaesthetic, are alternative pain treatments for patients where other analgesics and radiotherapy fail to give pain relief. Local anaesthetics lessen the need for opioids and thereby minimize opioid induced adverse effects. Motor paralysis of the lower extremities, which is a frequent side effect related to intrathecal or epidural administration of local anaesthetics, is usually avoided for pain indicating that the neuroaxial catheter is inserted in the upper part of the thoracic column. Still, epidural or intrathecal pain therapies are invasive, are associated with a risk for infections, and need very close follow-up (26).

Intra pleural analgesia that involves administering local anaethetics into the pleural space has been described and is felt to be effective for some malignancies (27). However, no data exists for this procedure in patients with MPM and, therefore, recommending such a treatment in this patient group is difficult.

\subsubsection{Cordotomy}

Despite all of the above interventions, many patients with MPM continue to suffer from severe pain. In these instances, there may be a role for percutaneous cervical cordotomy (PCC). This procedure interrupts the spinothalamic tracts at the level of $\mathrm{C} 1 / 2$ and causes loss of pain sensation contralaterally. Unfortunately, there are no prospective, randomised data on the role of cordotomy in MPM. The evidence comes from case series such as that by Jackson et al. They performed a retrospective review of 52 patients with MPM who underwent PCC. Their results showed that over $80 \%$ of patients were able to reduce their opioid requirements after the intervention and 38\% stopped opioids completely. At nine weeks post PCC, 18 patients had a recurrence in their pain requiring an increase in analgesia. Mild weakness was noted in four patients and dysaesthesia was noted in two patients. The authors concluded that PCC had a low complication rate and was successful in treating pain associated with MPM. A Turkish group reported on 165 patients who underwent PCC, 19 of whom had MPM (28). Of these 19 MPM patients, 13 were followed up for a median of 5.9 months with six patients being lost to follow up. The only complication reported was one case of post cordotomy dysaesthesia and all patients had an improvement in pain after the procedure. They recommend that all patients with local pain due to MPM should be considered for PCC.

\subsection{Conclusions}

The pain associated with MPM is extremely challenging to manage. Patients will often require a multitude of analgesic drugs since opioids alone are often insufficient to control the pain. It is not uncommon for patients to be on paracetamol, anti inflammatories, opioids, adjuvant analgesics in addition to topical treatments. For 
some, this combination of drugs may provide adequate analgesia. However, many patients continue to suffer from pain despite this cocktail of drugs. In these patients, other options should be considered. Chemotherapy, while offering a potential survival advantage, does not appear to have a significant impact on pain. Radiotherapy may be of benefit in some patients though prospective randomised data is lacking. Finally, again, despite a lack of prospective randomised data, neuroaxial pain therapy or cordotomy should be considered for patients whose pain is refractory to other treatments.

\section{Footnotes}

Authors' Contribution The algorithm was mainly done by JJ and LH, and reviewed and justified by JC. JJ produced the draft of the manuscript and JC produced the final version after edition and correction.

Financial Disclosure Teaching research project of education bureau of Anhui province, China (2014JYXM027) and key sciences research foundation of education bureau of Anhui province, China (SK2015A457) for the financial supports.

Funding/Support This work is supported by the teaching project of education bureau of Anhui province, China (2014JYZM027) and key sciences research foundation of education bureau of Anhui province, China (SK2015A457).

\section{References}

1. Davidson B. New diagnostic and molecular characteristics of malignant mesothelioma. Ultrastruct Pathol. 2008;32(6):227-40. doi: 10.1080/01913120802454298. [PubMed: 19117264].

2. Muers MF, Stephens RJ, Fisher P. Active symptom control with or without chemotherapy in the treatment of patients with malignant pleural mesothelioma (MS01): a multicentre randomised trial. Lancet. 2008;371:1685-94. doi:10.1016/S0140-6736(08)60727-8.

3. Jackson MB, Pounder D, Price C, Matthews AW, Neville E. Percutaneous cervical cordotomy for the control of pain in patients with pleural mesothelioma. Thorax. 1999;54(3):238-41. [PubMed: 10325900].

4. Salminen EK, Silvoniemi M, Syrjanen K, Kaasa S, Kloke M, Klepstad P. Opioids in pain management of mesothelioma and lung cancer patients. Acta Oncol. 2013;52(1):30-7. doi: 10.3109/0284186X.2012.725944. [PubMed: 23025295].

5. Geneva W. Cancer pain relief. World Health Organization; 1996.

6. Caraceni A, Hanks G, Kaasa S. Use of opioid analgesics in the treatment of cancer pain: evidenced-based recommendation from the EAPC. Lancet Oncol. 2012;13:58-68. doi:10.1016/S1470-2045(12)70040-2.

7. Donnelly S, Davis MP, Walsh D, Naughton M, World Health O. Morphine in cancer pain management: a practical guide. Support Care Cancer. 2002;10(1):13-35. [PubMed: 11777184].

8. Klepstad P, Kaasa S, Jystad A, Hval B, Borchgrevink PC. Immediateor sustained-release morphine for dose-finding during start of morphine to cancer patients: a randomised, double-blind trial. Pain. 2003;101:193-8. doi: 10.1016/S0304-3959(02)00328-7.
9. Gilron I, Bailey JM, Tu D, Holden RR, Weaver DF, Houlden RL. Morphine, gabapentin, or their combination for neuropathic pain. NEngl J Med. 2005;352(13):1324-34. doi: 10.1056/NEJMoa042580. [PubMed: 15800228].

10. Saarto T, Wiffen PJ. Antidepressants for neuropathic pain. Cochrane Database Syst Rev. 2007 doi:10.1002/14651858.cd005454.pub2.

11. Preskorn SH, Irwin HA. Toxicity of tricyclic antidepressants-kinetics, mechanism, intervention: a review. J Clin Psychiatry. 1982;43(4):151-6. [PubMed: 7068546].

12. Backonja MM. Anticonvulsants (antineuropathics) for neuropathic pain syndromes. Clin J Pain. 2000;16(2 Suppl):S67-72. [PubMed: 10870743].

13. Dworkin RH, O'Connor AB, Backonja M, Farrar JT, Finnerup NB, Jensen TS, et al. Pharmacologic management of neuropathic pain: evidence-based recommendations. Pain. 2007;132(3):237-51. doi: 10.1016/j.pain.2007.08.033. [PubMed: 17920770].

14. Freynhagen R, Strojek K, Griesing T, Whalen E, Balkenohl M. Efficacy of pregabalin in neuropathic pain evaluated in a 12-week, randomised, double-blind, multicentre, placebo-controlled trial of flexible- and fixed-dose regimens. Pain. 2005;115(3):254-63. doi: 10.1016/j.pain.2005.02.032. [PubMed: 15911152].

15. Mishra S, Bhatnagar S, Goyal GN, Rana SP, Upadhya SP. A comparative efficacy of amitriptyline, gabapentin, and pregabalin in neuropathic cancer pain: a prospective randomized double-blind placebo-controlled study. Am J Hosp Palliat Care. 2012;29(3):177-82. doi: 10.1177/1049909111412539. [PubMed: 21745832].

16. Bell RF, Eccleston C, Kalso E. Ketamine as adjuvant to opioids for cancer pain. A qualitative systematic review. J Pain Symptom Manage. 2003;26(3):867-75. [PubMed: 12967737].

17. Fleming JA, O'Connor BD. Use of lidocaine patches for neuropathic pain in a comprehensive cancer centre. Pain Res Manag. 2009;14(5):381-8. [PubMed: 19862373].

18. Jones VM, Moore KA, Peterson DM. Capsaicin $8 \%$ topical patch (Qutenza)-a review of the evidence. J Pain Palliat Care Pharmacother. 2011;25(1):32-41. doi: 10.3109/15360288.2010.547561. [PubMed: 21426216].

19. Scherpereel A, Astoul P, Baas P, Berghmans T, Clayson H, de Vuyst $\mathrm{P}$, et al. Guidelines of the European Respiratory Society and the European Society of Thoracic Surgeons for the management of malignant pleural mesothelioma. Eur Respir J. 2010;35(3):479-95. doi: 10.1183/09031936.00063109. [PubMed: 19717482].

20. Bissett D, Macbeth FR, Cram I. The role of palliative radiotherapy in malignant mesothelioma. Clin Oncol (R Coll Radiol). 1991;3(6):315-7. [PubMed: 1720658].

21. Jenkins P, Milliner R, Salmon C. Re-evaluating the role of palliative radiotherapy in malignant pleural mesothelioma. Eur J Cancer. 2011;47(14):2143-9. doi:10.1016/j.ejca.2011.05.012. [PubMed: 21658936].

22. van Meerbeeck JP, Gaafar R, Manegold C, Van Klaveren RJ, Van Marck EA, Vincent M, et al. Randomized phase III study of cisplatin with or without raltitrexed in patients with malignant pleural mesothelioma: an intergroup study of the European Organisation for Research and Treatment of Cancer Lung Cancer Group and the National Cancer Institute of Canada. JClin Oncol. 2005;23(28):6881-9. doi: 10.1200/JCO.20005.14.589. [PubMed:16192580].

23. Vogelzang NJ, Rusthoven JJ, Symanowski J, Denham C, Kaukel E, Ruffie $\mathrm{P}$, et al. Phase III study of pemetrexed in combination with cisplatin versus cisplatin alone in patients with malignant pleural mesothelioma. J Clin Oncol. 2003;21(14):2636-44. doi: 10.1200/JCO.2003.11.136. [PubMed: 12860938].

24. Bottomley A, Gaafar R, Manegold C, Burgers S, Coens C, Legrand C, et al. Short-term treatment-related symptoms and quality of life: results from an international randomized phase III study of cisplatin with or without raltitrexed in patients with malignant pleural mesothelioma: an EORTC Lung-Cancer Group and National Cancer Institute, Canada, Intergroup Study. J Clin Oncol. 2006;24(9):1435-42. doi: 10.1200/JCO.2005.03.3027. [PubMed: 16446322]. 
25. Chapman A, Mulrennan S, Ladd B, Muers MF. Population based epidemiology and prognosis of mesothelioma in Leeds, UK. Thorax. 2008;63(5):435-9. doi: 10.1136/thx.2007.081430. [PubMed: 18202164].

26. Christo PJ, Mazloomdoost D. Interventional pain treatments for cancer pain. Ann N Y Acad Sci. 2008;1138:299-328. doi: 10.1196/annals.1414.034. [PubMed: 18837908].

27. Amesbury B, O'Riordan J, Dolin S. The use of interpleural analgesia using bupivacaine for pain relief in advanced cancer. Palliat Med. 1999;13(2):153-8. [PubMed: 10474698].

28. Kanpolat Y, Savas A, Ucar T, Torun F. CT-guided percutaneous selective cordotomy for treatment of intractable pain in patients with malignant pleural mesothelioma. Acta Neurochir(Wien). 2002;144(6):5959. doi: 10.1007/s00701-002-0945-2. [PubMed: 12111493] discussion 599. 